\title{
Linking the Unitary Paradigm to Policy Through a Synthesis of Caring Science and Integrative Nursing (feature article)
}

\author{
Running Title: Linking the Unitary Paradigm to Policy \\ Mary S. Koithan, PhD, CNS-BC, FAAN 1 \\ Mary Jo Kreitzer, PhD, RN, FAAN ${ }^{2}$ \\ Jean Watson, PhD, RN, AHN-BC, FAAN ${ }^{3}$ \\ ${ }^{1}$ University of Arizona, Colleges of Nursing and Medicine \\ ${ }^{2}$ University of Minnesota, Center for Spirituality \& Healing and School of Nursing \\ ${ }^{3}$ Watson Caring Science Institute
}

\begin{abstract}
The principles of integrative nursing and caring science align with the unitary paradigm in a way that can inform and shape nursing knowledge, patient care delivery across populations and settings, and new healthcare policy. The proposed policies may transform our healthcare system in a way that supports nursing praxis and honors the discipline's unitary paradigm. This call to action provides a distinct and hopeful vision of a healthcare system that is accessible, equitable, safe, patient-centered, and affordable. In these challenging times, it is the unitary paradigm and nursing wisdom that offer a clear path forward.
\end{abstract}

Key Words: caring science, integrative nursing, policy, unitary paradigm

In 2014, the American Academy of Nursing became a founding member of the Nurses on Boards Coalition, a group of national nursing organizations working together to increase nurses' presence on corporate and non-profit health-related boards of directors throughout the country. The Academy did this, citing the need and potential contribution that nursing's expertise and authority on the patient experience, quality of care, and patient 
safety would bring to ensure the nation's health. With the goal of placing 10,000 nurses on boards of directors by 2020 and the incredible opportunities that we have to positively impact the healthcare system, we are left to ponder whether there is a guiding framework for these policy endeavors. What policy wisdom can be found within nursing's paradigmatic perspectives? Can our discipline's beliefs and values guide not only our clinical care but also the policies that could create a healthcare system that provides care that is culturally safe, personalized, and meaningful in a way that is financially responsible and responsive? This article addresses these questions from a unitary perspective of nursing by applying principles from integrative nursing and caring science to recommend policies for contemporary nursing practice.

\section{Nursing's Unitary Paradigm}

From its earliest beginnings, nursing has been a discipline focused on caring and healing from a holistic perspective, consistent with what we today generally refer to as the unitary paradigm. Florence Nightingale (1859/1946), often referred to as the founder of modern nursing, noted in the late 1800 s that the role of the nurse was to put the patient in the best possible condition so that nature could act and healing occur. This natural, healing link between the inherently whole person and environment, foundational in Nightingale's work, was an early expression of nursing's unitary paradigm, developed further in the writings of Martha Rogers and other nursing scholars.

While the essence of nursing has long been theorized from unitary paradigmatic views, contemporary nursing practice in many settings around the globe has become increasingly fragmented and de-stabilized. Nursing shortages in many parts of the world are significant, yet administrative bureaucracies often remove nurses from the point of care, be that the 
bedside, home, or clinic, replacing them with less skilled workers, shifting nursing responsibilities toward documentation and other administrative tasks. Technology, while lifesaving, also has the potential of creating distance between nurse and patient, particularly if the nurse attends more to the "machine" than the patient.

Patients tell this story from a different point of view. Many experience a long parade of "care providers" who are too busy to actually care. Or, they encounter different nurses on every shift day after day, making it very difficult to establish a relationship that builds trust and confidence. In many settings, care has become so fragmented that care coordinators or care managers are required to maintain some semblance of order.

We propose that together the principles of integrative nursing and caring science align with the unitary paradigm in a way that can practically shape nursing knowledge to inform the delivery of care across patient populations and clinical settings. Moreover, we posit that it is time to transform our healthcare system through policy initiatives that support praxis that honor nursing's unitary paradigm. In doing this, we recognize that it is challenging to change nursing practice until all relevant systems (legal/regulatory, financial, scientific, professional, ethical) re-envision health and well-being and the actions that support its attainment. This call to action provides a distinct and hopeful perspective in the ongoing throes of the national healthcare policy debate and offers nurses the opportunity to lead from a position of shared vision and common purpose.

\section{Caring Science: Defining the Moral and Ethical Basis of Patient-Centered Integrative Nursing}


As noted by Donaldson and Crowley (1978), nursing has a responsibility to continuously evolve its moral and ethical commitment to humanity. Since the 1970s, care and caring have been identified as foundational to nursing's covenant with society (Leininger, 1980; Watson, 1979). The American Nurses' Association, the American Association of Colleges of Nursing and the National League for Nursing have all identified caring as a foundational value for nursing. ANA (2015) describes caring as the "moral ideal of nursing, consisting of human-to-human attempts to protect, enhance, and preserve humanity and human dignity, integrity, and wholeness by assisting a person to find meaning in illness, suffering, pain, and existence" (p. 11). Caring, from a unitary perspective, is differentiated from emotion and benevolent wishes; rather caring is knowledge manifested as competent, sensitive, mature, skilled action that is grounded in science.

First described by Watson in 1979, caring science is based upon the Ethic of Belonging and the Ethic of Face developed by Emmanuel Levinas (1969), a French philosopher, who posited that our ethical worldview is the first principle of science. An Ethic of Belonging acknowledges that we all belong to the infinite field of universal cosmic love; we come from this source and will all ultimately return to it, manifesting the sacred circle of life-death. Thus, the following ideas apply:

- The universal whole (belonging) precedes our separate being;

- The human spirit is infinite;

- Consciousness - that awareness of the infinite source -- is unified and non-local.

- Our belonging ties us morally to the unfolding and continuous evolution of consciousness toward infinity. 
Translated, we conclude that an ethic grounded in caring means that "I should act in such a way never to treat the other person as a means to an end, but rather as an end in himself or herself. “

In the Ethic of Face, Levinas (1969) reminds us that our presence with "the other" in any caring-healing relationship is not merely physical or aesthetic. The face we encounter is authentic, raw, and vulnerable and is viewed as irreducible and without bounds, one in which we glimpse our own humanity. The "face" is not relegated to abstractions or ideations but is instead is experienced "as-lived" in its totality. In person-centered, relationship-based care, we are "invited" to "demand more of ourselves", accept our "obligation to demand justice" for the whole, and "commit ourselves to ensuring a meaningful world for the Other" (Levinas, 1969, pp. 66, 198, 201, 219). Therefore, when we see self as other and other as self, we acknowledge the depth of our shared humanity and "establish the primordial basis for our caring" and the unitary nature of our existence (Watson, 2008, p. 57). Through caritas, nursing commits to a practice that is precious and deeply meaningful; caring that is life-giving and life-receiving, affirming the discipline's unitary perspective.

\section{Caritas Processes ${ }^{\mathrm{TM}}$ : Foundation of Person-centered, Relationship-based Care}

Ten Caritas Processes ${ }^{\mathrm{TM}}$, informed by the values of caring science, operationalize nursing's moral-ethical commitment to person-centered, relationship-based care (Watson, 2008). Together, these processes convey how nurses enter into transpersonal caring moments with patients, families, and communities, and how we hold ourselves accountable to care that sustains the human bodymindspirit and supports the evolution of consciousness, locally and globally. They form the foundation of our practice. 
Therefore, nurses define person-centered care as heart centered; honoring and protecting the human dignity of the other and believing in their intrinsic value and worth. It is a relationship that is deeply respectful; we meet the other authentically and intentionally- human-to-human. We hold the space within which we build this relationship as sacred, reflective of the greater whole and the compassionate love that we all merit.

\section{Integrative Nursing: A Practice Model for Contemporary Healthcare Systems}

While caring science provides the moral-ethical foundations of nursing practice, integrative nursing offers direction that can inform and shape both practice and policy. At a time when nurses individually and nursing departments collectively are adopting caring science as their professional practice model, the principles of integrative nursing offer additional clarity about nursing's role in the delivery of care across patient populations and clinical settings to promote health and wellbeing.

Integrative nursing is described as a whole person/whole system way of beingknowing-doing that advances the health and wellbeing of persons, families and communities through caring/healing relationships (Kreitzer \& Koithan, 2014). Six principles guide the practice of integrative nursing [see Figure 1]. These are aligned with the unitary nature of human beings and healing, particularly as expressed in Watson's caring science. The Ethics of Belonging and Face inform integrative nursing's moral and ethical foundations (Principle 1) while the Caritas Processes describe the nature of personcentered, relationship-based care (Principles 2 and 4) and provide behavioral guidelines for the integrative nurse. Similarly, integrative nursing (Principles 3, 5, and 6) extends caring science and informs nursing action and policy in the current healthcare delivery 
system. Therefore, caring science encompasses the universals of human caring while integrative nursing encompasses the universals of human healing providing a paradigm for $21^{\text {st }}$ century nursing and healthcare.

\section{Figure 1. The Principles of Integrative Nursing}

1. Human beings are whole systems, inseparable from their environments.

2. Human beings have the innate capacity for health and wellbeing.

3. Nature has healing and restorative properties that contribute to health and wellbeing.

4. Integrative nursing is person-centered and relationship-based.

5. Integrative nursing practice is informed by evidence and uses the full range of therapeutic modalities to support/augment the healing process, moving from least intensive/invasive to more, depending on need and context.

6. Integrative nursing focuses on the health and wellbeing of caregivers as well as those they serve.

Source: Kreitzer, M.J. \& Koithan, M. (2014). Integrative nursing. New York: Oxford Press. Used with permission.

The 6 principles of integrative nursing are divided into two sets: The Foundations of Integrative Nursing, and Nursing Practice and Care Delivery. All of the principles are grounded in the unitary paradigm and align with Caring Science. The first set of 3 principles delineates foundational beliefs about human beings, their environment and 
health, and nursing practice. The second set of 3 principles focuses on integrative nursing and healing processes. Clinical manifestations and policy implications are associated with each of the 6 principles.

\section{The Foundations of Integrative Nursing}

The first three principles of integrative nursing are grounded in the unitary paradigm and align with particular Caritas Processes (CP). Beliefs underlying these principles range from statements about the nature of humans as being innately whole and healing, to the nature of nursing practice as person-centered and relationship-based. Policies derived from these principles emphasize caring practices that occur at the individual and interpersonal levels, but also include system-level practices.

\section{Human beings are whole systems inseparable from their environments.}

People are dynamic, individualistic and complex and as such, cannot be reduced to diagnoses, symptoms and deviations from norms. Caring for the "whole person" requires attentiveness to the indivisible nature of the person (bodymindspirit) and the inseparability of person from environment. The Ethic of Belonging situates this principle --morally and ethically. Integrative nurses view their patients and families as a reflection of self; rendering care with loving kindness (CP 1) and reverentially and respectfully assisting the patient to sustain human dignity (CP 9). Because human beings are complex adaptive systems, integrative nurses are open to the mystery of unfolding futures; we expect that outcomes can be unexpected, miracles do occur, and that local interventions often create change that is unanticipated (CP 10).

Clinical manifestations of these beliefs and values include the following: 
- Comprehensive assessment focused on bodymindspirit of the person as well as their environments and contexts.

- Personalized care that reflects the patient's and family's unique needs, strengths and preferences.

- A personalized care environment that is conducive to healing.

- Recognition that the nursing attitudes, actions, and body language are part of the healing environment.

Practicing nursing from a whole person/whole systems perspective begins with the recognition that the healthcare system must work for all people, regardless of socioeconomic, developmental, cultural, or physician needs and limitations. As such, ensuring health equity and adequate access to high quality, culturally safe, and affordable care becomes one of nursing's policy imperatives. In addition, the profession is called to create a system that is reflective of the whole population and to create educational programs that admit not only the so-called brightest students but those that can contribute to the wellbeing of our emerging social identity.

\section{Human beings have the innate capacity for health and wellbeing.}

The roots of this principle can be found across nursing theory beginning with Nightingale (1859/1946) who encouraged nurses to "assist the reparative process which Nature has instituted" (p. 6). Likewise, Parse (2015) claimed that health is a process of human enfolding/unfolding co-constituted with human-universe process. Reed (1997, cited in Reed, 2011) defined nursing "ontologically as inherent processes of well-being within and among human systems" (p. 16). The tenets of caring science propose that 
healing is a transformative process that occurs as the person recognizes the essential connectedness between self and universal consciousness.

Integrative nursing recognizes that the body has healing and restorative capacities on many levels. When the integrity of the skin is damaged by a cut, scrape or deeper wound, the body automatically goes into a process of inflammation, cell proliferation and ultimately cellular repair. While neurons do not divide and are not capable of mitosis after injury, surviving nerve cells, appropriately supported, reorganize and establish new neural connections. The brain's property of neuroplasticity allows for changes in its structure and function, a result of experiences as well as purely internal mental activity, our thoughts. Positive emotions flood our brains with dopamine and serotonin, enhance immune system functioning, diminish the inflammatory response to stress and change the scope and boundaries of the brain.

While recognizing that the capacity to heal is innate, integrative nurses also recognize that change is unpredictable and emergent. For example, the inflammatory response is helpful over a short period of time but when present over several weeks or months this same inflammatory process can produce a host of negative physiological changes. Integrative nursing practice seeks to reduce negative effects by providing the degree of support necessary to facilitate the innate healing process. For some patients, healing is supported by being authentically present and enabling faith/hope/belief (CP 2), clearly adopting the stance that the locus of healing is within the individual and not externally controlled. In other cases, support may require more intensive treatments that include hydration and medication administration. Always, integrative nurses are open to possibilities and embrace the mystery of the unknown to unfold and manifest (CP 10). 
Clinical manifestations of these beliefs and values include the following:

- A focused intention for healing and wholeness during care procedures.

- A nurturing and empowering presence with hope and belief in the agency of the patient/family/community.

- A focus on strengths, resources, and opportunities rather than problems and weaknesses.

When guided by the principle that healing is an innate capacity of the person, the healthcare system becomes supportive rather than directive. Consumers become active participants in their health rather than passive recipients of care. With this shift in focus, providers must have adequate time to coach and support an active patient and caregiving system. The primary care team will expand to include coaches, RN care coordinators, and individuals from other disciplines who can provide services that include stress reduction techniques, nutritional support, and lifestyle enhancement. Providers partner with individuals, families, and communities in roles and accountabilities that are greatly expanded. Health-related interventions move from prescription of medication to social interventions and societal transformation (e.g., transportation, housing, nutrition) as a first-line of support. Integrative nursing invites policy that recognizes the particular context (social, relational, temporal, cultural) and supports interventions that are affordable, feasible, and actively engage patients as partners.

\section{Integrative nursing is person-centered and relationship-based.}

Caring-healing relationships, characterized by empathy, caring, love, warmth, trust, confidence, credibility, honesty, kindness, respect and authentic communication, provide 
the context for integrative nursing. Person-centered care focuses on care of the whole person - bodymindspirit. Relationship-based care is built on continuity over time and calls upon the nurse to be fully present (CP 2), to listen deeply (CP 5), and to establish an authentic connection with the patient and family (CP 4). Providing care of this nature requires a deep knowledge and connection with those we serve as cultivated by listening to the other person's story (CP 5), staying within the other's frame of reference (CP 7), and creatively seeking solutions that honors the other (CP 6). Relationship-based care requires the integrative nurse to cultivate self-understanding and sensitivity to self in relationship through reflective practices. Reflection allows the nurse to discern when care is ego-centric and facilitates adoption of a more transpersonal, heart-centered, and emergent approach (CP 3).

Clinical manifestations of these beliefs and values include the following:

- Staff who know the patients' stories, contexts, and aspirations.

- Care that is individualized, anticipatory and based on patient/family needs and preferences.

- Staff who listen deeply: listens to learn, uses silence, and suspends judgment

- Staff and administration who value and practice reflectively.

- Staff who are concerned about each other, recognizing the whole persons they are working with.

Integrative nursing invites us to embrace the moral commitment that we live in right with each other, our communities, with the earth and its creatures, and with ourselves. Therefore, policy implications are wide-ranging; from organizational staff/scheduling patterns that lead to continuity of nurse/patient relationships, to support for a robust and 
active public health system that employs community health workers who can establish meaningful relationships within sociocultural environments of diverse populations. Integrative nurses are activists - recognizing that whole person wellbeing is intimately tied to the food security and safety of our communities, respect and personal freedom for individuals as well as the sovereign rights of indigenous nations, and our sense of space and place. Integrative nurses are committed to creating systems that are responsive, compassionate, and caring at both the individual and global levels of scale.

\section{Nursing Practice and Care Delivery}

Three additional principles of integrative nursing, grounded in the unitary view and consistent with caring science, provide the basis for developing policy that facilitates nursing praxis within contemporary healthcare systems. These principles extend our understanding of nursing interventions, nursing care delivery and health policy, aligning with current safety and quality initiatives. These initiative include the Quadruple Aim, the Magnet Recognition Program, and the Beacon Award for Excellence. They also reflect the public's interest in self-care, prevention, and less invasive therapeutic interventions and an increasing evidence base for a broad range of therapies and healing practices including mind-body techniques, body-based therapies, and natural products.

\section{Nature has healing and restorative properties that contribute to health and} wellbeing. 
According to the biophilia hypothesis, human beings are innately drawn to nature and the natural world, and nature has properties that are healing and restorative (Wilson, 1984). Recent systematic literature reviews support the growing evidence that being in nature is associated with reduction in blood pressure as well as reduced heart rate and respiratory distress/shortness of breath. Moreover, preliminary evidence is pointing to changes in biological markers associated with the stress response and changes in neurological activity and brain activation (Devlin \& Arneill, 2003; Ulrich, et. al., 2008). Recognizing the healing power of nature, evidence-based design of healthcare facilities is incorporating elements of biophilic design, including the use of natural light, color, art and architectural features, such as curves, that mimic nature. There is significant growth in nature-based therapeutics, including the use of labyrinths, healing gardens, animal-assisted interactions and facilitated green exercise.

Clinical manifestations of these beliefs and values include the following:

- Clinical settings that provide access to natural light.

- Clinical settings that integrate intentional use of color, art, and music that connect patients, families, and staff to nature-based environments.

- Interventions (e.g., guided imagery, meditations, movement therapies) that draw upon nature.

- Clinical settings that use of labyrinths and healing gardens and spaces.

Once again, nurses are called to extend their policy commitments and activism beyond what has historically been considered mainstream health-related policies. Policies based drawing from this integrative nursing principle would be designed to protect our natural resources, ensure biodiversity, and guarantee that food supplies are devoid of chemicals 
and modifications harmful to human and animal systems. We would remember that our wellbeing is intricately linked to the multitude of systems that surround us and that all of us thrive when we have access to natural light, green environs, and adequate whole foods. Local policy implications include advocacy for the arts in schools, support for public art, and promotion of responsive rather than functional architecture (Beesely, et. al., 2009).

\section{Integrative nursing is informed by evidence and uses the full range of therapeutic modalities to support/augment the healing process, moving from least intensive and invasive to more, depending on need and context.}

Over the past 20 years, there has been significant growth in the use of integrative therapies and healing practices (Nguyen, et. al., 2011; Okoro, et. al., 2013). The drivers of this global phenomenon are many and include the limitations of western medical approaches in managing symptoms, particularly of chronic disease, and the desire of people to use non-pharmacologic approaches to improve their health and wellbeing. Many of the so-called integrative therapies fall within the scope of nursing practice and in the U.S., some state nurse licensing boards have developed specific statements that acknowledge the use of integrative therapies.

Clinical manifestations of these beliefs and values include the following:

- Interventions that are based on a patient's condition (depth of distress and dysfunction), needs, wants and preferences.

- Providers (nurses, physicians, therapists, ancillary staff) who employ a full range of therapeutics and healing practices to manage symptoms, improve clinical outcomes, and quality of life. 
A major focus in integrative nursing is the effective management of symptoms, acknowledging the role of the nurse as supportive of the innate healing process, aligning nursing's science and philosophical beliefs and values with its practice. Conventional biomedical management of symptoms frequently begins with a pharmacological intervention intended to suppress symptoms and "fix" the problem, with the locus of healing often on the provider of care. Integrative nursing shifts the focus from curing to healing, changing the nature of problem solving and prioritizing. This does not imply that biomedical interventions are discarded. Rather they are introduced when that level of intervention is warranted and the system condition requires more intensive support. Nurses practicing from an integrative perspective are not merely "adding on" integrative therapies; integrative therapies become core to their practice.

Cutshall and Van Getson (2014) describe the evidence underlying the use of integrative therapies for the management of nausea that includes dietary interventions, aromatherapy oils (ginger and peppermint), mind-body approaches (guided imagery, relaxation, hypnosis and deep breathing), acupressure (P6 acupressure point), and energy healing such as Reiki. Non-pharmacologic pain management approaches include mind/body interventions such as the relaxation response, guided imagery and mindfulness-based stress reduction as well as acupressure and acupuncture, yoga and movement therapies, massage, and access to nature (Wagner \& Thompson, 2014). Integrative approaches are also described for the management of stress, sleep, anxiety, depressed mood, fatigue, cognitive impairment and care of the human spirit.

Policy implications suggested by this principle are wide-ranging to include agencybased protocols that facilitate the use of the full range of therapeutics, and delineate 
methods of integrative assessment and clinical decision-making. Policies such as these would enable a broad range of providers to practice to the full scope of their education, licensure, and certification. Healthcare team membership and conceptions of interprofessional practice would expand in response to policy implications of this principle; professional credentialing and privileging would be extended to include Traditional Chinese Medicine providers, massage therapists, healing touch practitioners, and others. Reform would stretch across the insurance and reimbursement systems to appropriately pay for therapies that have been deemed both effective and safe by an expanded definition of evidence that includes multiple ways of knowing.

Consider for one moment the potential impact of policy reforms suggested by this single principle alone. How many iatrogenic conditions could we prevent if we could simply teach children to treat pain using hot/cold before reaching for medication? What might be the long-term, downstream financial, social, and physiological effects when an entire generation believes in and expects to be able to address common symptoms through selfcare and low-cost therapies rather a neighborhood pharmacy and a pill? How much could we save if we tried manipulative, body-based therapies for recurrent headaches before we demanded an MRI and extensive neurological testing? What impact would we have on cost, quality of life, and productivity if we encouraged people to participate in stress-reduction activities rather than asking for the latest prescription advertised on television?

Integrative nursing invites us to return to a system grounded in our roots - a system focused on "putting the patient in the best condition for nature to act", a system that is accessible, patient/family-centered, and culturally safe. It is a system that embraces health for all --- including its caregivers. 


\section{Integrative nursing focuses on the health and wellbeing of caregivers as well}

\section{as those they serve.}

Nurses work in intense, high-stress environments and are vulnerable to stress and burnout that impact their own health and wellbeing as well as their care of patients. Caring science emphasizes that nursing is essentially a reciprocal process where nurse and patient are in mutual relationship. This reciprocity requires that nurses attend to their own needs; the nurse needs to take care of self in order to be able to care for others (Watson, 2001).

Self-awareness and self-care are core practices foundational to integrative nursing. Self-awareness allows the nurse to notice inner experiences while caring for patients. Selfreflection, a form of ongoing inquiry, can lead to deeper learning and insights. Self-care is the most sustainable healthcare practice and comprises attentiveness to lifestyle behaviors (including healthy eating, exercise, sleep and stress management) and may include the use of integrative therapies such as meditation, yoga, energy therapies and massage.

Clinical manifestations of these beliefs and values include the following:

- Staffing patterns that reflect a value of provider self-care, allowing for adequate break times, staffing support, and reflective practice.

- Staff development and on-boarding processes that provide for reflective practice skill development.

Policies based upon this principle would require work environments to incorporate self-care settings and services (e.g., wellness centers, mind-body and movement classes, spas) for staff. Staff would be supported in developing and implementing personal plans 
for health and wellbeing. Employee policies might include clinical ladders and promotion and tenure criteria that reward engagement in self-care and lifestyle change.

\section{Conclusion: Blueprint for Practice and Policy}

The convergence of caring science and integrative nursing advances our understanding of nursing's metaparadigm and provides a blueprint for a practice grounded in the unitary paradigm. The message here is consistent with the historic call for nursing to come of age as a mature discipline and profession of caring, healing, and health. It is reflective of our emphasis on caring presence during the human health experience. By aligning the moralethical foundations provided by caring science and the Caritas Processes with an integrative practice model, we provide a practical approach for nursing in the 21st century. This unified approach honors nursing's unitary paradigm while clearly charting the course for the discipline's policy agenda.

\section{References}

American Nurses Association. (2015). Nursing: Scope and standards of practice (3rd ed.). Silver Spring, MD: ANA.

Beesley, P., Hirosue, S., Ruxton, J., Trankle, M., \& Turner, C. (Eds.) (2006). Responsive architectures: Subtle technologies. Riverside, CA: Riverside Architectural Press.

Cutshall, S. \&Van Getson, L. (2014). Integrative nursing management of nausea. In M. J. Kreitzer \& M. Koithan, (Eds.). Integrative Nursing (pp. 221-236). New York: Oxford Press.

Devlin, A. \& Arneill, A. (2003). Health care environments and patient outcomes. Environment and Behavior, 35(5), 665-694.

Donaldson S. \& Crowley, D. (1978). The discipline of nursing. Nursing Outlook, 26(2), 113120. 
Koithan, M. (2015). Gazing with soft eyes: Envisioning a responsive integrative healthcare system. Global Advances in Health and Medicine, 4(3), 7-8.

Kreitzer, M. J. (2015). Integrative nursing: Application of principles across clinical settings. Rambam Maimonides Medical Journal, 6(2), 1-8.

Kreitzer, M.J. \& Koithan, M. (2014). Integrative nursing (Eds.). New York: Oxford Press.

Leininger, M. (1980). Caring: A central focus for nursing and health care services. Nursing and Health Care, 1(10),135-143, 176.

Levinas E. (1969). Totality and infinity: An essay on exteriority (A. Lingis, Trans.) Pittsburgh: Duquesne University Press.

Nguyen, L., Davis, R., Kaptchuk, T. \& Phillips, R. (2011). Use of complementary and alternative medicine and self-rated health status: results from a national survey. Journal of General Internal Medicine, 26(4), 399-404.

Nightingale, F. (1859/1946). Notes on nursing. London: Harrison and Sons.

Okoro, C., Zhao, G., Li, C. \& Balluz, L. (2013). Has the use of complementary and alternative medicine therapies by U.S. adults with chronic disease-related functional limitations changed from 2002 to 2007? Journal of Alternative and Complementary Medicine, 19(3), 217-223.

Parse, R.R. (2015). Rosemarie Rizzo Parse's humanbecoming paradigm. In M.C. Smith \& M.E. Parker (Eds.), Nursing theories \& nursing practice (4th ed.), (pp. 263-277).

Philadephia: F.A. Davis.

Reed, P.G. (2011). The spiral path of nursing knowledge. In P.G.Reed \& NB.C Shearer, Nursing knowledge and theory innovations: Advancing the science of practice (pp. 1-36). New York: Springer.

Ulrich, R., Zimring, C., Zhu, X., DuBose, J., Seo, H. \& Choi, Y. (2008). A review of the research literature on evidence- based healthcare design. Health Environments Research and Design Journal, 1(3), 61-125.

Wagner, J. \& Thompson, S. (2014). Integrative nursing management of pain. In M.J. Kreitzer \& M. Koithan, (Eds.) Integrative Nursing (pp. 292-306). New York: Oxford University Press.

Watson, J. (1979). Nursing: The philosophy and science of caring. Boston: Little Brown.

Watson, J. (2001). Post-hospital nursing: Shortages, shifts, and scripts. Nursing Administration Quarterly, 25(3), 77-82. 
Watson, J. (2008). Nursing: The philosophy and science of caring. Boulder: University of Colorado Press.

Wilson, E.O. (1984). Biophilia: The human bond with other species. Cambridge: Harvard University Press. 